\title{
ESTABILIDADE OXIDATIVA DE ÓLEOS VEGETAIS UTILIZADOS EM FRITURAS DE MANDIOCA PALITO CONGELADA ${ }^{1}$
}

\author{
Mara da Silva CORSINI ${ }^{2, *}$, Neuza JORGE ${ }^{2}$
}

\section{RESUMO}

O presente estudo teve como objetivo determinar a medida da estabilidade oxidativa e a alteração total dos óleos de algodão, girassol e palma durante a fritura descontínua de mandioca palito congelada. As frituras foram conduzidas em uma fritadeira elétrica doméstica, onde o óleo foi aquecido, à temperatura de $180^{\circ} \mathrm{C}$, por $25 \mathrm{~h}$, com reposição de óleo fresco. Para a análise da estabilidade oxidativa das amostras de óleos, obtidas durante as frituras, foram realizadas a medida do período de indução (horas) e determinação de compostos polares totais (\%). Os resultados mostraram uma diminuição do período de indução, independente do tipo de óleo. O óleo de palma refinado apresentou um maior período de indução quando comparado ao óleo de algodão e girassol, o que pode ser atribuído à diferença da composição em ácidos graxos. Todos os óleos em estudo apresentaram valores de compostos polares totais abaixo do limite de descarte para óleos de fritura, exigido pela legislação internacional (24-27\%). Verificou-se também que, à medida que se formaram os compostos de degradação, diminuiu a estabilidade oxidativa dos óleos.

Palavras-chave: lipídios, fritura, mandioca palito congelada, estabilidade oxidativa.

\section{SUMMARY}

OXIDATIVE STABILITY OF VEGETABLE OILS USED IN FROZEN CASSAVA CHIPS FRYING. The present study aimed at determining the oxidative stability measurement and the total alteration of cotton, sunflower and palm oils during frozen cassava chips discontinued frying. The fryings were performed in a household electric frying pan, where the oil was heated up to $180^{\circ} \mathrm{C}$ temperature, for $25 \mathrm{~h}$, with fresh oil replacement. For the oxidative stability analysis of the oil samples obtained during the fryings, were used the induction period (hours) and total polar compounds determinations (\%). The results showed a decrease in the induction period, regardless of the oil type. The refined palm oil presented a higher induction period when compared to the cotton and sunflower oils, which can be attributed to the fatty acids composition difference. All of the oils studied showed total polar compounds values beneath the discard limit for frying oils ruled by the international legislation (24-27\%). It was also noticed that, as the degradation compounds were formed, the oils oxidative stability decreased as a result.

Keywords: lipids, deep fat frying, frozen cassava chips, oxidative stability.

\section{1 - INTRODUÇÃO}

A aceitação de alimentos processados por fritura é universal e apreciada por diferentes grupos populacionais. A fritura de alimentos consiste simplesmente em introduzir o alimento em um banho de óleo ou gordura que se encontra em elevada temperatura. No entanto, esta técnica culinária, que parece tão simples, é um processo extraordinariamente complexo, em que se encontra envolvida uma grande quantidade de fatores [7].

Alguns destes fatores que intervêm são dependentes do próprio processo como o tipo de equipamento utilizado, a temperatura, o tempo e o método de fritura. Outros, extrínsecos ao mesmo, se relacionam com o tipo de óleo utilizado, sua composição, sua relação superfície/volume, suas características físico-químicas, presença de aditivos, contaminantes, etc., assim como a natureza do

\footnotetext{
${ }^{1}$ Recebido para publicação em: 11/6/2004. Aceito para publicação em 23/1/2006 (001360)

${ }^{2}$ Instituto de Biociências, Letras e Ciências Exatas. Universidade Estadual Paulista (Unesp)

Rua Cristóvão Colombo, 2.265 - Jardim Nazareth

CEP 15054-000 - São José do Rio Preto (SP)

E-mail:maracorsini@yahoo.com.br

*A quem a correspondência deve ser enviada
}

alimento, peso/volume, tipo de preparação: empanados, pré-fritos [21].

O óleo é um dos componentes mais críticos em um sistema de fritura. A sua degradação depende da maior ou menor presença de ácidos graxos insaturados em sua composição. Óleos vegetais que possuem uma grande quantidade de ácidos graxos poliinsaturados estão mais sujeitos à oxidação do que óleos que possuem maior quantidade de ácidos graxos saturados [13].

O tempo de aquecimento do óleo influencia na quantidade de compostos de alteração formados durante a fritura. O sistema intermitente de aquecimento das frituras descontínuas é muito mais destrutivo para os óleos e gorduras de frituras que no sistema de aquecimento contínuo. Uma das explicações atribui este efeito ao fato de que, em elevada temperatura, as reações oxidativas ocorrem fundamentalmente na superfície de contato com o ar; enquanto durante o resfriamento, ao diminuir a velocidade das mesmas e aumentar a solubilidade do ar, favorece a entrada de ar na massa, produzindo maior quantidade de hidroperóxidos e radicais livres durante o posterior aquecimento [21].

Muitos experimentos mostraram que óleos mantidos em uso contínuo apresentaram melhor qualidade final do 
que aqueles que sofreram processo descontínuo, tanto quando se manteve a temperatura constante e alta, como sob oscilações [17, 23]. Este fato se deve porque, nas frituras contínuas, o vapor formado recobre a superfície do óleo formando uma barreira isolante entre o óleo da superfície e o oxigênio do ar.

$\mathrm{O}$ alimento também exerce efeito sobre o processo e a qualidade do óleo por meio de diversos fatores envolvidos nele: sua composição, seu tamanho e a forma como foi preparado [19]. As diversas mudanças registradas nas atitudes do consumidor ao longo dos últimos anos fizeram com que as indústrias de alimentos passassem a dispor de produtos específicos para o processo de fritura, como os alimentos congelados, que são largamente aceitos pelo mercado consumidor.

A maioria dos trabalhos envolvendo processos de fritura utiliza a batata como alimento, mas, recentemente, foi lançada no mercado a mandioca palito congelada, um produto ainda pouco estudado no Brasil, principalmente durante processos de fritura.

Como resultado das frituras, transformações são produzidas nas propriedades físico-químicas e sensoriais do alimento. A fritura confere ao alimento características agradáveis de cor, sabor, textura e palatabilidade. Entretanto, podem ocorrer também reações que modificam as qualidades funcionais e nutricionais dos alimentos, podendo chegar a níveis em que não se consegue mais produzir alimentos de qualidade [15, 20].

Durante o processo de fritura de imersão, os óleos são continuamente expostos à ação de vários fatores que levam a uma grande diversidade de reações químicas, tais como: hidrólise, oxidação e polimerização da molécula [22].

A perda da estabilidade oxidativa de um óleo se deve às reações de oxidação dos lipídios. A oxidação é um processo degradativo que ocorre quando o oxigênio atmosférico ou aquele que está dissolvido no óleo reage com ácidos graxos insaturados presentes. As reações químicas envolvidas no processo de oxidação dos óleos são muito complexas e geram, em seus estágios mais avançados, produtos sensorialmente inaceitáveis [12].

O processo de oxidação pode ser favorecido e intensificado pela incidência de luz, que atua como catalisador. Os ácidos graxos insaturados são mais sensíveis à oxidação do que os saturados. As gorduras que tenham sofrido processo de oxidação tendem a escurecer, aumentar a viscosidade, incrementar a formação de espumas e desenvolver sabor e aromas indesejáveis [18].

O objetivo da determinação dos compostos polares é separar a amostra de óleo, com base em sua diferente polaridade, utilizando a cromatografia de adsorção, em duas frações. Uma fração, a fração não polar, contém a gordura que permanece sem alteração ou triglicerídios não alterados e, na segunda fração, ou fração polar, se concentram os compostos de degradação ou compostos polares. O que dá idéia do nível global de alteração ou da alteração total produzida pelas diferentes variáveis envolvidas neste processo [21].

Devido à complexidade do processo, não há um único método analítico que possa detectar todas as situações que envolvem a deterioração de óleos no processo de fritura. A determinação dos compostos polares totais por cromatografia em coluna tem sido reportada por vários autores como um dos melhores métodos da determinação do estado de alteração do óleo de fritura [4, 5]. Existem também alguns métodos de avaliação da estabilidade oxidativa que monitoram as alterações ocorridas na amostra, mediante análises como índice de peróxidos, análise sensorial, determinação de dienos conjugados, valor de carbonila, análise de voláteis, como Rancimat e Oil Stability Instrument [1].

É necessário entender as mudanças e as alterações que os óleos vegetais sofrem durante períodos longos de aquecimento e buscar critérios objetivos para definir quando os óleos devem ser descartados. Isso pode levar a otimização dos processos de fritura e, conseqüentemente, garantir produtos de melhor qualidade nutricional.

O presente estudo teve como objetivo determinar a medida da estabilidade oxidativa e a alteração total dos óleos de algodão, girassol e palma durante a fritura descontínua de mandioca palito congelada.

\section{2 - MATERIAL E MÉTODOS}

Para os ensaios de fritura descontínua de mandioca palito congelada, in natura, foram utilizados o óleo de algodão refinado (OAR), óleo de girassol refinado (OGR) e óleo de palma refinado (OPR).

Para a operação de fritura foi utilizada uma fritadeira elétrica, marca NKS home - modelo DF-150/AL, com capacidade total de 1,5 L, com temperatura controlada a $180^{\circ} \mathrm{C} \pm 5^{\circ} \mathrm{C}$. Além do próprio termostato da fritadeira, a temperatura do óleo foi monitorada com o auxílio de termômetro. Seguindo as características do processo de fritura conforme Tabela 1, 50 lotes de mandioca, com aproximadamente 250 g/lote foram fritos, por $5 \mathrm{~h} /$ dia, durante cinco dias consecutivos.

TABELA 1 - Características do processo de fritura

\begin{tabular}{lc}
\hline Condições do processo & OAR, OGR e POR \\
\hline Tipos de óleo & Mandioca palito congelada \\
Produto & $1,5 \mathrm{~L}$ \\
Quantidade de óleo na fritadeira & $250 \mathrm{~g}$ \\
Quantidade de produto a ser frito & $1: 6$ \\
Proporção de alimento/óleo & $0,2 \mathrm{~cm}^{-1}$ \\
Relação superfície/volume & $180^{\circ} \mathrm{C} \pm 5^{\circ} \mathrm{C}$ \\
Temperatura de fritura & $5 \mathrm{~h}$ \\
Tempos de fritura/dia & $25 \mathrm{~h}$ \\
Tempos de fritura total & 10 \\
Número de frituras/dia & 50 \\
Quantidade total de frituras & \\
\hline
\end{tabular}


Ao final de cada dia, foi realizada a filtragem do óleo, com o auxílio de um Passador Chinoy, para remoção de resíduos formados. Durante o processo de fritura descontínua foram empregados intervalos de $30 \mathrm{~min}$, sendo $5 \mathrm{~min}$ para o reaquecimento do óleo.

As amostras de óleo e mandioca foram coletadas em diferentes intervalos de tempo: amostra inicial, ao final da primeira e a cada cinco frituras, ou seja, nos tempos $0 ; 0,5 ; 2,5 ; 5 ; 7,5 ; 10 ; 12,5 ; 15 ; 17,5 ; 20 ; 22,5$ e $25 \mathrm{~h}$. As amostras com $50 \mathrm{~mL}$ de óleo foram colocadas em vidro âmbar e acondicionadas em congelador a $-18^{\circ} \mathrm{C}$, para evitar qualquer alteração oxidativa até a realização das análises.

O reabastecimento com óleo novo foi realizado sempre que necessário para compensar perdas resultantes da absorção pelo alimento durante a fritura e manter constante a relação superfície/volume $\left(0,2 \mathrm{~cm}^{-1}\right)$ da fritadeira. A adição total de óleo novo foi de $1.650 \mathrm{~mL}$ para o óleo de algodão, $1.800 \mathrm{~mL}$ para o óleo de girassol e $1.850 \mathrm{~mL}$ para o óleo de palma.

Para a medida da estabilidade oxidativa foi utilizado o método proposto pela AOCS [2]. O equipamento Rancimat, marca Metrohm, modelo 743, foi utilizado para realização das análises, nas seguintes condições: $3 \mathrm{~g}$ de óleo, temperatura da análise em $100^{\circ} \mathrm{C}$ e fluxo de ar em $20 \mathrm{~L} / \mathrm{h}$. Uma curva de condutividade elétrica $\mathrm{x}$ tempo é automaticamente plotada com o decorrer da reação e do teste, e o período de indução é determinado em horas.

A determinação de compostos polares totais mediante cromatografia em coluna foi obtida conforme o método cromatográfico proposto por DOBARGANES, VELASCO, DIEFFENBACHER [9].

Os resultados obtidos das determinações analíticas foram submetidos às análises de variância para determinar a influência dos fatores sobre a alteração dos óleos aquecidos a elevadas temperaturas, em duas repetições. $\mathrm{O}$ experimento foi realizado em um esquema fatorial $3 \mathrm{x}$ 12, com 3 tipos de óleos e 12 tempos de fritura, no delineamento inteiramente casualizado [11]. As análises de variância e os testes de Tukey para as médias foram obtidos por meio do programa Estat (Sistema para Análises Estatísticas), versão 2.0.

\section{3 - RESULTADOS E DISCUSSÃO}

A composição em ácidos graxos dos óleos iniciais utilizados no presente estudo indicou diferenças entre eles (Tabela 2). Observou-se que os óleos de algodão e girassol apresentaram maiores valores para ácidos graxos poliinsaturados, sendo 52,80 e 60,67\%. Por outro lado, o óleo de palma apresentou em sua composição um maior valor para ácidos graxos saturados (48,21\%). Verificou-se também que o óleo de palma apresentou um maior valor de ácidos graxos monoinsaturados $(42,39 \%)$ quando comparado com os óleos de algodão e girassol, cujos valores encontrados foram 16,78 e $27,23 \%$, respectivamente.
TABELA 2 - Caracterização dos óleos iniciais utilizados no processo de fritura de mandioca palito congelada

\begin{tabular}{lccc}
\hline Ácidos graxos (\%) & OAR & OGR & OPR \\
\hline Saturados & 28,04 & 11,03 & 48,21 \\
Monoinsaturados & 16,78 & 27,23 & 42,39 \\
Poliinsaturados totais & 52,80 & 60,67 & 8,89 \\
Ômega 3 & 0,12 & 0,19 & 0,22 \\
Trans & $<0,01$ & $<0,01$ & $<0,01$ \\
NI & 2,36 & 1,08 & 0,55 \\
$\begin{array}{l}\text { Índice de peróxidos (meq/kg) } \\
\text { Dienos conjugados (\%) }\end{array}$ & 3,20 & 3,40 & 0,5 \\
$\begin{array}{l}\text { OAR=óleo de algodão refinado, OGR=óleo de girassol refinado, OPR=óleo de palma } \\
\text { refinado, NI=não identificado }\end{array}$ & 1,33 & 0,51 & 0,28 \\
\end{tabular}

refinado, $\mathrm{NI}=$ não identificado

Para o índice de peróxidos, notou-se que os valores das amostras iniciais encontraram-se dentro das margens permitidas pelas normas que regulamentam a adequação de um óleo inicial para o consumo no Brasil (Resolução $n^{\circ} 482 / 99$ - Anvisa), que estabelece para os óleos de algodão, girassol e palma refinados $10 \mathrm{meq} / \mathrm{kg}$ para índice de peróxidos [3]. Em relação aos dienos conjugados, notouse, para os óleos iniciais, uma maior porcentagem para o óleo de algodão refinado (1,33\%), seguido pelos óleos de girassol $(0,51 \%)$ e palma $(0,28 \%)$. Observou-se ainda, que dentre os óleos estudados, o óleo de palma foi o que apresentou, inicialmente, o menor índice de peróxidos e a menor porcentagem de dienos conjugados.

Após realizada as análises de variância para o período de indução e determinação de compostos polares totais, utilizando os valores obtidos ao longo do período experimental de 25 h, observou-se que para as duas determinações, que o teste $\mathrm{F}$ foi significativo $(\mathrm{P}<0,01)$ para os efeitos principais e para a interação óleos $x$ tempos de fritura, sendo, então, necessário proceder ao desdobramento dessa interação cujos resultados encontram-se na Tabela 3.

A estabilidade oxidativa está intimamente relacionada com o grau de insaturação do óleo. Observou-se, para os óleos iniciais, um menor período de indução para o óleo de palma (141,34 h) e menor para o óleo de girassol (10,43 h). Como a autoxidação é um processo que ocorre entre o oxigênio molecular e os ácidos graxos insaturados, era de se esperar que o período de indução do óleo de palma, mais saturado, fosse bem superior ao dos óleos de algodão e girassol, mais insaturados.

Verificou-se, na Tabela 3, que os valores do período de indução diminuíram ao longo do tempo de fritura, independentemente do tipo de óleo. Em relação aos tempos de fritura para cada óleo, observou-se, para o óleo de algodão refinado, que os tempos de fritura 0 e 0,5 h não diferiram entre si e diferiram significativamente dos demais tempos estudados. Para o óleo de girassol refinado, notou-se que os valores do período de indução mantiveram-se praticamente 
constante, não havendo diferença significativa ao longo dos tempos de fritura. Verificou-se, para o óleo de palma, que a partir de $5 \mathrm{~h}$ de fritura os valores não diferiram significativamente entre si.

Quanto aos óleos dentro de cada tempo de fritura, observou-se que óleo de palma refinado apresentou, em todos os tempos de fritura, maior período de indução. Verificou-se também que os resultados do óleo de palma diferiram significativamente dos valores encontrados para os demais óleos na maioria dos tempos de fritura.

DEL RÉ [8], estudando o comportamento dos óleos vegetais em frituras descontínuas, utilizando dois produtos distintos (batata palito pré-frita congelada e produto cárneo empanado pré-frito congelado - snacks), verificou que a estabilidade oxidativa durante a fritura de batatas foi menor que dos snacks, devido à quantidade de ácidos graxos saturados liberados no óleo durante a fritura do produto cárneo empanado, reforçando que a estabilidade oxidativa está igualmente relacionada com o grau de insaturação do óleo ou gordura.

Segundo LUMLEY [14], o conteúdo de compostos polares totais em um óleo novo deve oscilar entre $0,4 \mathrm{e}$ $6,4 \%$. De acordo com os resultados obtidos para compostos polares totais, observou-se, primeiramente, que os valores das amostras iniciais, para os três óleos, encontraram-se dentro dos limites estabelecidos para óleos refinados. Verificou-se também que as amostras iniciais dos óleos de algodão e palma apresentaram valores superiores ao encontrado para o óleo de girassol, provavelmente devido ao processamento industrial.

A determinação da quantidade total dos produtos de alteração, originados como conseqüência do processo, constitui a base das limitações de uso dos óleos existentes em alguns países, estabelecida em torno de $24-27 \%$ de compostos polares [10].

Considerando o limite de descarte acima estabelecido, verificou-se, por meio da Tabela 3, que os óleos estudados apresentaram valores bem abaixo deste limite, sendo o maior valor médio encontrado, $15,16 \%$, para a fritura com o óleo de algodão refinado.

Alguns autores atribuem os baixos valores de compostos polares à constante reposição de óleo novo empregado nestes processos [5, 6, 8, 16]. Neste trabalho, a reposição total com óleo novo, necessária para restituir o óleo absorvido pelo alimento e, assim, manter constante a relação superfície/volume, contribuiu para os baixos níveis de compostos polares totais.

Observou-se, por meio da Tabela 3, que a porcentagem de compostos polares totais aumentou gradativamente com o decorrer dos tempos de fritura para os óleos de algodão e girassol. Notou-se que a formação dos compostos polares foi bem maior para o óleo de girassol do que para o óleo de algodão. Para o óleo de palma refinado, verificou-se um comportamento instável ao longo dos tempos de fritura, oscilando entre um aumento e uma diminuição.

Quanto aos óleos dentro de cada tempo de fritura, observou-se que a partir de 7,5 h de fritura os valores de compostos polares totais dos óleos de algodão e girassol não diferiram significativamente entre si, e ambos diferiram dos valores encontrados para o óleo de palma. Este fato pode estar atribuído à diferença do grau de insaturação dos óleos analisados.

Dentre os óleos estudados, verificou-se que o óleo de palma refinado apresentou, ao longo do tempo de fritura, menor formação de compostos polares e, conseqüentemente, maior estabilidade oxidativa.

Com a proposta de comparar os métodos aplicados foram determinados os coeficientes de correlação entre os testes analíticos realizados para cada óleo de fritura, considerando os três tipos de óleos e os 12 tempos de fritura. Verificou-se um baixo índice de correlação $\left(-0,58^{* *}\right)$ devido, provavelmente, à grande heterogeneidade das amostras. Com isso, os coeficientes de correlação entre as determinações analíticas foram determinados separa-

TABELA 3 - Desdobramento da interação óleos $x$ tempos de fritura para os dados do período de indução e de compostos polares totais

\begin{tabular}{|c|c|c|c|c|c|c|c|c|c|c|c|c|}
\hline Óleos & \multicolumn{12}{|c|}{ Tempos de fritura (h) } \\
\hline \multicolumn{13}{|c|}{ Período de indução (h) } \\
\hline OAR & $26,17^{\text {ав }}$ & $21,97^{\mathrm{aB}}$ & $14,52^{\mathrm{bB}}$ & $12,39^{\mathrm{bB}}$ & $12,29^{\mathrm{bB}}$ & $11,97^{\text {bв }}$ & $12,26^{\mathrm{bB}}$ & $11,20^{\text {ьв }}$ & $11,02^{\mathrm{bB}}$ & $10,59^{\text {вв }}$ & $10,43^{\text {ьв }}$ & $10,49^{\mathrm{bB}}$ \\
\hline OGR & $10,43^{\mathrm{ac}}$ & $8,90^{\mathrm{ac}}$ & $7,55^{\mathrm{ac}}$ & $7,60^{\mathrm{ac}}$ & $7,41^{\mathrm{aC}}$ & $7,75^{\mathrm{aB}}$ & $7,06^{\mathrm{aC}}$ & $7,74^{\mathrm{aB}}$ & $7,33^{\mathrm{aB}}$ & $7,28^{\mathrm{aB}}$ & $7,51^{\mathrm{aB}}$ & $7,30^{\mathrm{aB}}$ \\
\hline \multicolumn{13}{|c|}{ Compostos polares totais (\%) } \\
\hline OAR & $5,32^{\mathrm{fA}}$ & $5,85^{\mathrm{fA}}$ & $7,05^{\mathrm{efA}}$ & $9,40^{\mathrm{deA}}$ & $10,64^{\mathrm{cdA}}$ & $11,79^{\mathrm{bcdA}}$ & $12,64^{\mathrm{bcA}}$ & $12,97^{\mathrm{abcA}}$ & $13,06^{\mathrm{abcA}}$ & $14,21^{\mathrm{abA}}$ & $14,04^{\mathrm{abA}}$ & $15,17^{\mathrm{aA}}$ \\
\hline OGR & $2,41^{\mathrm{fB}}$ & $3,38^{\mathrm{fB}}$ & $5,96^{\mathrm{eAB}}$ & $7,43^{\mathrm{deB}}$ & $9,55^{\mathrm{cdA}}$ & $10,33^{\mathrm{bcA}}$ & $11,86^{\mathrm{abcA}}$ & $12,12^{\mathrm{abA}}$ & $13,54^{\mathrm{aA}}$ & $13,66^{\mathrm{aA}}$ & $13,57^{\mathrm{aA}}$ & $14,20^{\mathrm{aA}}$ \\
\hline OPR & $5,32^{\mathrm{bA}}$ & $4,88^{\mathrm{bAB}}$ & $5,26^{\mathrm{bB}}$ & $5,64^{\mathrm{bc}}$ & $5,79^{\mathrm{bB}}$ & $6,57^{\mathrm{abB}}$ & $6,57^{\mathrm{abB}}$ & $8,75^{\mathrm{aB}}$ & $6,22^{\mathrm{bB}}$ & $5,74^{\mathrm{bB}}$ & $6,13^{\mathrm{bB}}$ & $6,85^{\mathrm{abB}}$ \\
\hline
\end{tabular}

OAR - óleo de algodão refinado, OGR=óleo de girassol refinado, POR - óleo de palma refinado. a, b - (linha) em cada óleo, médias dos tempos de fritura seguidas de mesma letra minúscula, não diferem entre si pelo teste de Tukey $(\mathrm{P}>0,05)$. A, B - (coluna) - em cada tempo de fritura, médias dos óleos seguidas de mesma letra maiúscula, não diferem entre si pelo teste de Tukey $(\mathrm{P}>0,05)$ 
damente para cada tipo de óleo e os 12 tempos de fritura, conforme apresentado na Tabela 4.

TABELA 4 - Coeficientes de correlação entre os testes analíticos para cada tipo de óleo

\begin{tabular}{lcc}
\hline Tipo de óleo & PI & CPT \\
\hline $\begin{array}{l}\text { Óleo de algodão } \\
\text { PI }\end{array}$ & 1,00 & \\
CPT & $-0,87^{* *}$ & 1,00 \\
Óleo de girassol & & \\
$\quad$ PI & 1,00 & 1,00 \\
CPT & $-0,79^{* *}$ & \\
Óleo de palma & & \\
$\quad$ PI & 1,00 & 1,00 \\
$\quad$ CPT & $-0,60^{*}$ & \\
\hline $\begin{array}{l}\text { PI }=\text { período de indução, CPT }=\text { compostos polares totais. }{ }^{* *} \text { teste significativo }(\mathrm{P}<0,01) \\
\text { *teste significativo }(\mathrm{P}<0,05)\end{array}$
\end{tabular}

Verificou-se que, quando estabelecidos por tipo de óleo, os coeficientes de correlação linear entre os métodos analíticos melhoraram. Dentre os tipos de óleos, observouse que o óleo de algodão apresentou melhor coeficiente de correlação $\left(-0,87^{* *}\right)$. Os valores de correlação inversa entre o período de indução e a análise de compostos polares totais demonstraram que à medida que se formam compostos de degradação, diminui a estabilidade oxidativa dos óleos.

\section{4 - CONCLUSÕES}

A estabilidade oxidativa dos óleos em estudo diminuiu ao longo dos tempos de fritura. Verificou-se que a estabilidade oxidativa do óleo de palma, mais saturado, foi bem superior à dos óleos de algodão e girassol, mais insaturados.

Os óleos de algodão, girassol e palma apresentaram baixos valores de compostos polares totais, não ultrapassando os limites recomendados por alguns países para descarte de óleos e/ou gorduras.

Observou-se que o óleo de palma refinado apresentou maior estabilidade e menor formação dos compostos polares. Entretanto, apesar das diferenças na composição em ácidos graxos, os óleos estudados não apresentaram valores acima dos limites recomendados em alguns países para o descarte dos mesmos, independentemente do tempo de aquecimento. Isto sugere que as condições estabelecidas no processo de fritura: tempo e temperatura de aquecimento, relação superfície/volume e reposição de óleo novo são seguras, garantindo produtos de qualidade e indicando que todos os óleos foram adequados à fritura.

\section{5 - REFERÊNCIAS BIBLIOGRÁFICAS}

[1] ANTONIASSI, R. Métodos de avaliação da estabilidade oxidativa de óleos e gorduras. Boletim do Centro de Pesquisa e Processamento de Alimentos, Curitiba, v. 19, n. 2, p. 353-380, 2001.

[2] AOCS. Official Methods and Recommended Practices of the American Oil Chemists' Society. Champaign: AOCS, 1993.
[3] BRASIL. Resolução n $^{\circ} 482$, de 23 de setembro de 1999. Regulamento técnico para fixação de identidade e qualidade de óleos e gorduras vegetais. Diário Oficial da União, Brasília (DF), v. 196. Seção I, p. 82-87. 13 out., 1999.

[4] CELLA, R.C.F.; REGITANO-D'ARCE, M.A.B.; SPOTO, M. H. F. Comportamento do óleo de soja refinado utilizado em fritura por imersão com alimentos de origem vegetal. Ciência e Tecnologia de Alimentos, Campinas, v. 22, n. 2, p. 111-116, 2002.

[5] CUESTA, C.; SÁNCHEZ-MUNIZ, F.J. Quality control during repeated fryings. Grasas y Aceites, Sevilla, v. 49, n. 3-4, p. 310-318, 1998.

[6] DAMY, P. C. Alterações físico-químicas de óleo de soja e da gordura hidrogenada durante o processo de fritura descontínua de batatas. 2001. 136 f. Dissertação (mestrado em Engenharia e Ciência de Alimentos). Instituto de Biociências, Letras e Ciências Exatas. Universidade Estadual Paulista, São José do Rio Preto (SP).

[7] DAMY, P.C.; JORGE, N. Determinações físico-químicas do óleo de soja e da gordura vegetal hidrogenada durante o processo de fritura descontínua. Brazilian Journal of Food Tecnology, Campinas, v. 6, n. 2, p. 251-257, jul-dez, 2003.

[8] DEL RÉ, P.V. Comportamento de óleos vegetais em frituras descontínuas de produtos pré-fritos congelados. 2003. 121 f. Dissertação (mestrado em Engenharia e Ciência de Alimentos). Instituto de Biociências, Letras e Ciências Exatas - Universidade Estadual Paulista, São José do Rio Preto (SP).

[9] DOBARGANES, M.C.; VELASCO, J.; DIEFFENBACHER, A. Determination of polar compounds, polymerized and oxidized triacylglycerols, and diacylglycerols in oils and fats. Pure and Applied Chemistry, Oxford, v. 72, n. 8, p. 1.563-1.575, 2000.

[10] FIRESTONE, D.; STIER, R.F.; BLUMENTHAL, M.M. Regulation of frying fats and oils. Food Technology, Chicago, v. 45, n. 2, p. 90-94, 1991.

[11] GOMES, F.P. Curso de estatística experimental., 14 ed. Piracicaba, Editora Nobel, 477 p. 2000.

[12] LIMA, J.R.; GONÇALVES, L.A.G. Avaliação analítica de óleos utilizados em processo de fritura. Boletim da Sociedade Brasileira de Ciência e Tecnologia de Alimentos, Campinas, v. 29, n. 2, p. 186-192, 1995.

[13] LOLOS, M.; OREOPOULOU, V.; TZIA, C. Oxidative stability of potato chips: effect of frying oil type, temperature and oxidants. Journal of Science of Food and Agriculture, [S.1.], v. 79, p. 1.524-1.528, 1999.

[14] LUMLEY, I.D. Polar compounds in heated oils. In: VARELA, G.; BENDER, A.E.; MORTON, I.D. (Ed.). Frying of Foods: Principles, Changes, New Approaches. Chichester: Ellis Horwood, p. 166-173. 1988.

[15] MÁRQUEZ-RUIZ, G.; PÉREZ-CAMINO, M.C.; DOBARGANES, M.C. Evaluación nutricional de grasas termoxidadas y de frituras. Grasas y Aceites, Sevilla, v. 41, p. 432-439, 1990.

[16] MASSON, L. et al. Comportamiento de aceites poliinsaturados en la preparación de patatas fritas para consumo inmediato: Formación de nuevos compuestos y comparación de métodos analíticos. Grasas y Aceites, Sevilla, v. 48, n. 5, p. 273-281, 1997.

[17] MEHTA, U.; SWINBURN, B. A review of factors affecting fat absorption in hot chips. Critical Reviews in 
Food Science and Nutrition, Boca Raton, v. 41, n. 2, p. 133-154, 2001.

[18] MONFERRER, A.; VILLALTA, J. La fritura desde un punto de vista práctico (I). Alimentación, Equipos y Tecnologia, [S.1.], v. 21, n. 3, p. 85-90, 1993.

[19] PÉREZ-CAMINO, M.C. et al. Alteración de grasas usadas en fritura. II. Variables que influyen en el proceso en continuo y análisis real en freidoras industriales. Grasas y Aceites, Sevilla, v. 39, n. 1, p. 39-43, 1988.

[20] POTTEAU, B. et al. Recherches sur la composition et les effects physiologiques de l'huile de soja chauffès et de differentes fractions obtenues à partir de cette huile. Revue Française des Corps Gras, Paris, v. 25, p. 234-245, 1978.

[21] POZO-DÍEZ, R.M. Estudio del proceso de fritura de alimentos frescos y congelados prefritos. Comportamiento del aceite de semilla de girasol de alto contenido en ácido oleico. 1995, 338 f. Tese (doutorado em Farmacia). Facultad de Farmacia. Universidad de Alcalá de Henares, Alcalá de Henares.

[22] SANIBAL, A.A.E.; MANCINI-FILHO, J. Alterações físicas, químicas e nutricionais de óleos submetidos ao processo de fritura. Food Ingredients South American, São Paulo, v. 18, p. 64-71, mai-jun, 2002.

[23] STEVENSON, S.G.; VAISEY-GENSER, M.; ESKIN, N.A.M. Quality control in the use of deep frying oils. Journal of the American Oil Chemists' Society, Chicago, v. 61, n. 6, p. 1.102-1.108, 1984.

\section{6 - AGRADECIMENTOS}

À Companhia Refinadora da Amazônia (Agropalma), localizada em Belém (PA), pela doação do óleo de palma refinado e à empresa DeMarch Ind. e Com. de Frutas Ltda., localizada em Jundiaí (SP), pelo fornecimento da mandioca palito congelada. 